\title{
Plasmapheresis in Neurological Disorders
}

\author{
Francisco Tomaz Meneses de Oliveira and Charles Peter Tilbery \\ Department of Neurology, Neurologist, Santa Casa of São Paulo-SP, Brazil \\ "Corresponding author: Oliveira FTM, Department of Neurology, Santa Casa of São Paulo-SP, Brazil, CEP 01221-010, Tel: 551121767000; E-mail: \\ towmaz@gmail.com
}

Received date: October 24, 2016; Accepted date: December 06, 2016; Published date: December 12, 2016

Copyright: @ 2016 Oliveira FTM, et al. This is an open-access article distributed under the terms of the Creative Commons Attribution License, which permits unrestricted use, distribution, and reproduction in any medium, provided the original author and source are credited.

\section{Short communication}

During the history of medicine, the specialty of Neurology was, sometimes, stigmatized or labelled as a science of brilliant diagnoses, but reserved prognosis and limited treatments. Today, it is known that the technological development has changed this scenario, largely, due to spectacular advances in studies of molecular medicine, with excellent methods of diagnostic through imaging, molecular biology, muscle biopsy with electron microscopy, single fiber electromyography, digital electroencephalogram, video-electroencephalogram and more recently with the large number of antibodies [1].

Due to a large number of neurological syndromes associated with antibodies with increasing identification, it's been observed new potential therapeutic targets for neurological diseases, which they were formerly considered progressive diseases, degenerative or purely psychiatrics [1].

Today, there is a solid concept accepted by the international community and the literature that there are several neurological disorders of the central nervous system (CNS) and the peripheral nervous system (PNS) mediated by antibodies and they have several therapeutic strategies, with increasing options depending about the immune mechanism involved. Among the immunotherapy strategies for such diseases, we can use: corticosteroids (methylprednisolone, prednisone), immunosuppressant's (azathioprine, methotrexate, mycophenolate mofetil, cyclosporine, cyclophosphamide), monoclonal antibodies (natalizumab, rituximab, ocrelizumabe), anti-TNF alpha drugs (infliximab, adalimumab), intravenous Immunoglobulin and Plasmapheresis or Apheresis therapy, among many other options (many then already validated by multicentre randomized studies, other phase III studies, and some then established by usage for experts physicians based on the understanding of the pathophysiology of the diseases) $[1,2]$.

The immune-mediated neurological involvement occurs in a variety of ways: isolated CNS involvement (as in autoimmune Encephalitis, Multiple Sclerosis, Optic Neuromyelitis), SNP (chronic inflammatory demyelinating polyradiculoneuropathy, Guillain Barret syndrome, Multifocal Motor Neuropathy), neuromuscular junction (Myasthenia gravis), muscle (idiopathic inflammatory myopathies), simultaneous involvement of CNS and PNS (Morvan syndrome). In Morvan syndrome, it's identified the same antibody (anti-CASPR2, proteins present in VGKC complex-voltage-gated potassium channels) causing an acquired neuromyotonia (condition caused by hyper excitability of the peripheral nerve, causing twitching, cramps) and limbic encephalitis (immune-mediated behavioural, cognitive, psychiatric, epileptic and movement disorders secondary to involvement of the limbic system). [1]

Neoplasms were found in different proportions in each of these diseases, ranging from less than $10 \%$ of the cases up to about $50 \%$.
Thymoma is the most common. In 2007, antibodies against NMDA receptors (NR1 mainly) were identified commonly in young patients, especially women and young children who have a severe form of encephalitis that results in psychiatric disorders and movement disorders. In these cases, ovarian teratoma was neoplasia commonly found in adult women but rare in children $[1,2]$.

Other antibodies have recently been discovered, directed to a specific receptor or ion channel associated with a protein. Antibodies against glycine receptors were associated with a form of stiff syndrome (Stiff Person Syndrome), usually called Progressive Encephalomyelitis with rigidity and myoclonus. It is a condition which is well described in the literature, previously it lacked a proposal therapeutically effective, and it has now been recognized in many patients, with a broader range of clinical symptoms that may help in early and reliable clinical diagnosis. Use of corticosteroids, plasmapheresis and intravenous immunoglobulin are the best treatments $[1,3]$.

Several studies have demonstrated significant improvement in symptoms with the use of plasmapheresis in immune-mediated neurological diseases. The clinical decision is unique, according to availability and adverse effects profile of these treatments $[1,3]$.

Plasmapheresis is a procedure which is used therapeutically or just to collect material for transfusion. When therapeutic, it separates the patient's blood components replacing the plasma removed from a donor fluid, colloid or crystalloid, usually albumin or saline solution $[2,3]$. Historically, large amounts of plasma could only be exchanged via manual phlebotomy followed by centrifugation, a slow and complicated technique, which generally only allowed an exchange of $500 \mathrm{ml}$ per session [3]. It is today a highly complex procedure, often available only in reference centers.

The availability allows the physician can offer the patient an effective proven treatment of high impact from the clinical point of view. The pathogenic substance is an autoantibody, circulating immune complexes, lipoproteins, endotoxins, among others. The molecule large and long half-life for a faster removal than its endogenous clearance, and it's acutely resistant to conventional therapies so that the procedure is suitably indicated [4]. The most common adverse effect is hemodynamic instability. A careful evaluation of impact of this procedure recommended before treatment with clinical and laboratory tests. There are various immune-mediated neurological pathologies belong to the group of diseases in which plasmapheresis indicated [5].

In the article: "Plasmapheresis therapy for immune-mediated diseases in neurology: literature review", we can find a good review of the topic discussed, focusing on therapy indications with plasmapheresis or apheresis therapy [6].

Morgan et al. identified the neurological diseases considered of great response to the use of therapeutic apheresis (Optic 
Neuromyelitis, anti-MuSK positive Myasthenia Gravis, acute disseminated encephalomyelitis- ADEM, anti-NMDA encephalitis, Multiple Sclerosis relapsing-remitting, Polymyositis) and with high chance of new advances in therapeutic response in the next 5 to 10 years. Most neurological disease treatments with beneficial use of therapeutic apheresis (TA) are mediated by a humoral immune response, and these beneficial effects probably occur through the removal of pathogenic autoantibodies and associated inflammatory mediators [7-10].

The good therapeutic response is most often observed in diseases with an acute course against those of chronic evolution. This is probably secondary to the slow equilibrium between vascular and interstitial spaces, which influences in a complete removal of antibodies unlikely in chronic cases. The rate of antibodies removal by TA may express a first order reaction with respect to the peripheral blood; however, in the CNS this is not a rule because it involves more complex mechanisms [11-13].

The issue of ideal time for initiation of TA in relation to the development of symptoms is an important factor. TA is often an option after other treatments such as steroids in high doses. Besides, a major obstacle in achieving experimental works for further clinical studies is that there are no TA animal models to test hypotheses and so it's necessary to rely on human studies. Whereas many neurological conditions are rare, this becomes even more difficult to study [11-13].

In the last 5 years, significant advances have been made in the development and study of alternatives beyond TA, including IVIG and monoclonal antibodies (e.g., rituximab), so many studies will emerge in the coming years in relation to immunomodulatory therapies, demonstrating the importance of being up to date and to knowing each therapeutic modality [11-13].

\section{References}

1. Vincent A (2014) Autoimmune Encephalitis. J Neurol Neurosurg Psychiatry 85: e3.
2. Cortese I, Chaudhry V, So YT, Cantor F, Cornblath DR, et al. (2011) Evidence-based guideline update: Plasmapheresis in neurologic disorders: report of the Therapeutics and Technology Assessment Subcommittee of the American Academy of Neurology. Neurology 76: 294-300.

3. Linker C (1983) Plasmapheresis in clinical medicine. West J Med 138: 60-69.

4. Fridey JL, Kaplan AA (2014) Therapeutic apheresis: indications and technique. Up To Date 1-15.

5. Weinstein $\mathrm{R}$ (2008) Therapeutic apheresis in neurological disorders: a survey of the evidence in support of current category I and II indications for therapeutic plasma exchange. J Clin Apher 23: 196-201.

6. Oliveira FTM, Luca NC, Tilbery CP (2016) Plasmapheresis therapy for immune-mediated diseases in neurology: literature review. Global Vaccines Immunol 1: 29-32.

7. Morgan SM, Shaz BH, Pavenski K, Meyer EK, Delaney M, et al. (2014) The top clinical trial opportunities in therapeutic apheresis and neurology. J Clin Apher 29: 331-335.

8. Lehmann HC, Hartung HP, Hetzel GR, Stuve O, Kieseier BC (2016) Plasma exchange in neuroimmunological disorders: Part 1: rationale and treatment of inflammatory central nervous system disorders. Arch Neurol 63: 930-935.

9. Gwathmey K, Balogun RA, Burns T (2014) Neurologic indications for therapeutic plasma exchange: 2013 update. J Clin Apher 29: 211-219.

10. Mühlhausen J, Kitze B, Huppke P, Müller GA, Koziolek MJ (2015) Apheresis in treatment of acute inflammatory demyelinating disorders. Atheroscler 18: 251-256.

11. Kaya E, Keklik M, Sencan M, Yilmaz M, Keskin A, et al. (2013) Therapeutic plasma exchange in patients with neurological diseases: multicenter retrospective analysis. Transfus Apher Sci 48: 349-352.

12. Sorgun MH, Erdogan S, Bay M, Ayyildiz E, Yucemen N, et al. (2013) Therapeutic plasma exchange in the treatment of neuroimmunologic disorders: review of 92 cases. Transfus Apher Sci 49: 1741-1780.

13. Szczepiorkowski ZM, Winters JL, Bandarenko N, Kim HC, Linenberger ML, et al. (2010) Apheresis applications committee of the American Society for Apheresis. Guidelines on the use of therapeutic apheresis in clinical practice-evidence-based approach from the apheresis applications committee of the American society for apheresis. J Clin Apher 25: 83-177. 